Revue des patrimoines

37 | 2018

Jardins collectifs : de l'abbé Lemire aux jardins d'insertion. Typologies - Expériences - Enjeux de conservation

\title{
Les jardins partagés en Finlande
}

Community gardens in Finland

\section{Hanna Sorsa}

\section{(2) OpenEdition}

\section{Journals}

Édition électronique

URL : http://journals.openedition.org/insitu/19294

DOI : 10.4000/insitu. 19294

ISSN : 1630-7305

\section{Éditeur}

Ministère de la culture

Référence électronique

Hanna Sorsa, "Les jardins partagés en Finlande », In Situ [En ligne], 37 | 2018, mis en ligne le 13 décembre 2018, consulté le 01 mai 2019. URL : http://journals.openedition.org/insitu/19294 ; DOI 10.4000/insitu.19294

Ce document a été généré automatiquement le 1 mai 2019.

\section{(ब) $\Theta$}

In Situ Revues des patrimoines est mis à disposition selon les termes de la licence Creative Commons Attribution - Pas d'Utilisation Commerciale - Pas de Modification 4.0 International. 


\title{
Les jardins partagés en Finlande
}

Community gardens in Finland

\author{
Hanna Sorsa
}

\section{Introduction}

1 «Le paysage détient les archives les plus denses, le moindre fragment témoigne. » ${ }^{1}$

2 Avec l'urbanisation et l'augmentation de la densité de population dans les villes, les jardins partagés ont été créés pour fournir des ressources vivrières pour les plus pauvres, puis pour la classe moyenne, avant de se transformer en lieux de détente et de loisirs vers la fin $\mathrm{du} \mathrm{xx}^{\mathrm{e}}$ siècle. Leur utilisation, leur construction et leur conservation sont aujourd'hui encadrées par plusieurs réglementations en vigueur.

\section{Le contexte}

$3 \mathrm{Au}_{\mathrm{XIX}} \mathrm{e}^{\mathrm{s}}$ siècle, les espaces verts conçus aux abords des villes étaient intégrés à la politique d'urbanisation. Les jardins ouvriers étaient essentiellement des potagers. Ils gagnent un statut très important au xx $x^{e}$ siècle, pendant deux guerres : la guerre civile de 1928 et la guerre d'Hiver, en 1939, pour des raisons de pénurie de nourriture. La culture du jardin s'est étendue aux classes ouvrières au XIX ${ }^{\mathrm{e}}$ siècle. Les jardins privés ont été inspirés par l'art des jardins des manoirs et des forges. Les parcelles ont été aménagées tout d'abord pour leur fonction, puis pour leur esthétique. Les éléments décoratifs ont fait partie des jardins à partir des années $1930^{2}$. Vingt ans plus tard, les jardins étaient devenus une partie intégrante des maisons individuelles appelées « rintamamiestalo », maisons typiques en bois, construites après la guerre d'Hiver et comportant un étage et demi.

4 La Finlande a célébré les 100 ans de son indépendance en 2017. La plupart des influences en matière de jardins sont arrivées en Finlande à partir des autres pays scandinaves, notamment la Suède et le Danemark. Les Finlandais eux-mêmes ont beaucoup voyagé à l'étranger pour en importer des formes et des pratiques paysagères. La Finlande étant un 
pays éloigné du reste de l'Europe, les voyages à l'étranger y ont été considérés comme des démarches d'ouverture, facteur d'enrichissement culturel pour ce pays nordique.

\section{Les recherches récentes}

Figure 1

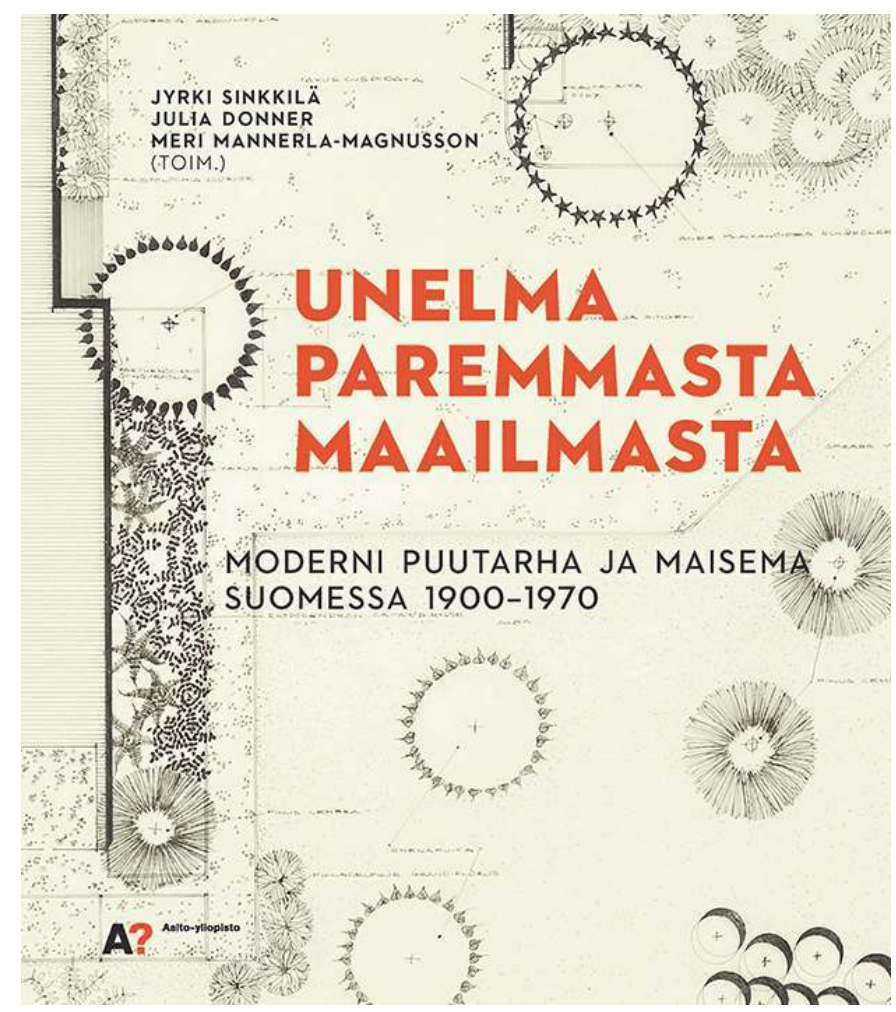

SINKKILÄ, Jyrki, DONNER, Julia, MANNERLA-MAGNUSSON, Meri (éd.). Unelma paremmasta maailmasta. Moderni puutarha ja maisema Suomessa 1900-1970. Helsinki : Aalto Arts Books, 2016.

5 La recherche sur l'histoire des jardins et du paysage est devenue un sujet important à partir des années 2000 en Finlande. Un certain nombre d'ouvrages ont été publiés. Ces recherches allient la sociologie, l'histoire et la philosophie et s'ajoutent aux inventaires historiques des sites paysagers et des villes. Le thème des jardins et des parcs est resté longtemps marginal dans les études universitaires. Toutefois, en une décennie, à l'intérêt général pour la nature en ville succèdent des recherches dans divers domaines universitaires (fig. 1). Une seule monographie est publiée en 2015 sur les jardins familiaux ${ }^{3}$. Les autres ouvrages sur ce thème orientés vers le grand public sont des guides, des livres de photographie ou de beaux livres (fig. 2). En l'absence d'informations de fond, le patrimoine des jardins familiaux n'est pas toujours bien connu et identifié, ni maintenu et préservé. 
Figure 2

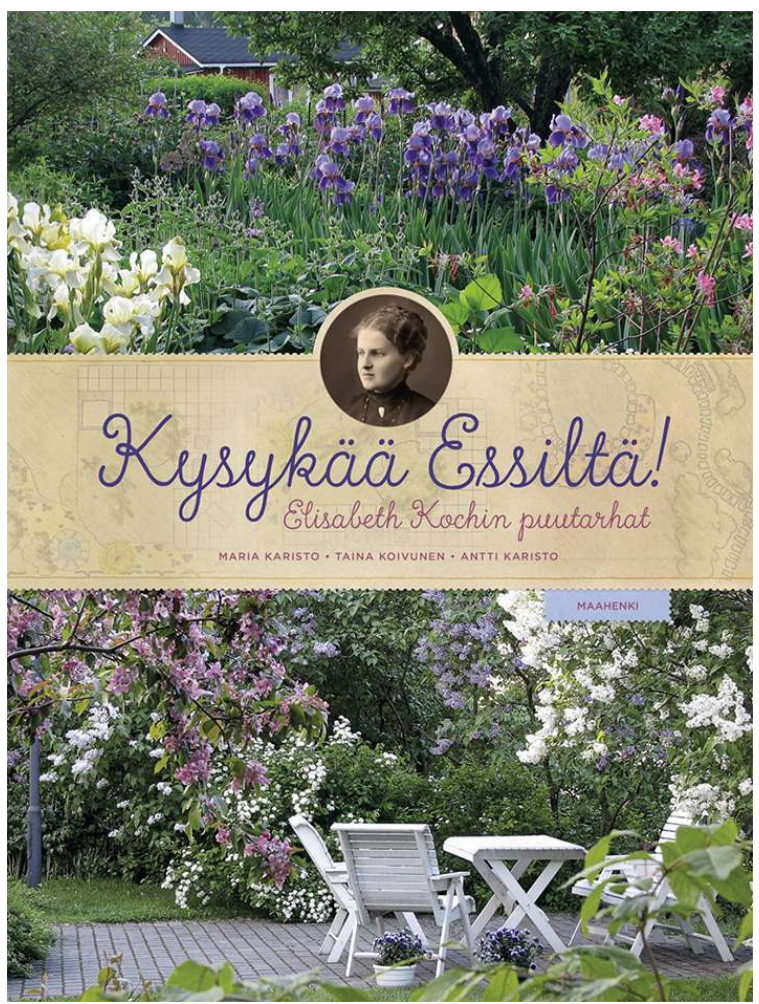

KARISTO, Maria, KOIVUNEN, Taina, KARISTO, Antti. Kysykää Essiltä! Elisabeth Kochin puutarhat. Maahenki : 2015.

6 «Comment pouvons-nous obtenir de l'information sur les parcs historiques et le paysage et que faire de cette information? $»^{4}$. Certains livres ont été édités pour aider à répondre à cette question. Ranja Hautamäki a édité un guide d'inventaire des parcs historiques intitulé Portti puutarhaan («Passerelle vers le jardin ») $)^{5}$. Elle y donne les grandes lignes pour constituer un inventaire des parcs et jardins. Le service des travaux publics de la Ville d'Helsinki a également publié un inventaire historique des parcs et sites paysagers ${ }^{6}$.

7 Le changement de fonction et les divers besoins de l'aménagement urbain ont détruit des entités paysagères importantes. La sensibilisation à l'histoire des jardins et des parcs, en particulier chez les étudiants en histoire, en architecture, en paysage, en sociologie, entre autres, est importante car c'est le meilleur moyen de comprendre l'histoire des lieux, de ces formes spécifiques et uniques, en un mot le génie d'un lieu. Les parcs et les jardins, leurs utilisateurs, familles, individus et associations, en tant qu'entités complexes, combinent l'histoire économique, sociale et artistique. À l'avenir, la conciliation de zones agricoles avec les nouvelles et anciennes parties des villes ouvrira des perspectives intéressantes, tant dans les études sur l'agriculture urbaine et la nature en ville que dans les autres études anthropologiques. De ce côté, les études sur les jardins familiaux et les parcelles urbaines se rejoignent.

8 Au travers des publications sur les jardins familiaux, on peut noter deux approches différentes: l'approche humaniste, qui parle de la pratique et du plaisir de jardiner ensemble, et l'approche institutionnelle, qui se concentre sur l'évolution et la compréhension du contexte sociologique et historique des jardins familiaux. Pour donner quelques exemples parmi les dernières études sur ces jardins, la paysagiste Maria Karisto 
a publié un livre sur le travail d'Elisabeth Koch (2015), la paysagiste Gretel Hemgård sur le jardin pédagogique de Kumpula à Helsinki (2016) et Mirja-Riitta Siivonen, Pirkko Salonen et Tuija Kuchka sur les jardins collectifs (1999). Ces articles illustrent les caractéristiques et la problématique de la conservation des jardins familiaux du point de vue historique. Ils présentent également les paysagistes, humanistes et jardiniers qui ont été à l'initiative des jardins partagés, sans oublier de rendre compte des enjeux spécifiques liés au maintien de ce patrimoine vivant.

\section{Études de cas}

9 En Finlande, la culture des jardins familiaux se divise en jardins familiaux, en finnois « siirtolapuutarha», jardins communautaires «yhteisöviljely» ou parcelles urbaines « palstaviljely».

10 Les jardins familiaux sont d'une surface moyenne de $300 \mathrm{~m}^{2}$, essentiellement situés sur des terrains affermés. $95 \%$ sont la propriété foncière des communes et seulement $5 \%$, de propriétaires fonciers privés. Les jardins familiaux forment des sites clos qui couvrent approximativement 300 hectares au total, divisés en parcelles de 250 à $500 \mathrm{~m}^{2}$. Il est possible de construire une « maisonnette » sur la parcelle et d'y planter des légumes, des fruits et des fleurs. Les parcelles urbaines sont par contre divisées en 1 à 100 lopins. Le propriétaire du terrain peut être la municipalité, l'Église ou un particulier. Les parcelles sont parfois louées à des familles. Les parcelles urbaines se différencient des jardins communautaires car chaque parcelle est à l'image de son propriétaire - il n'existe pas de règles qui limitent l'expression personnelle ou l'esthétique de l'aménagement de la parcelle ${ }^{7}$.

\section{Jardins familiaux}

\section{Historique}

11 La création des premiers jardins familiaux a été laborieuse. La pénurie alimentaire, après la Première Guerre mondiale, a accéléré la mise en place des "champs des pauvres ", terrains destinés à produire de la nourriture, principalement des pommes de terre. Les autres légumes étaient inconnus du peuple 8 . En fait, la terre avait été offerte au peuple uniquement pour cultiver des pommes de terre, et sans avoir le droit d'y construire. Cette culture de la première nécessité a été perçue comme un devoir et non comme une activité récréative. Le premier jardin familial a été fondé en 1915 à Hatanpää à Tampere par E. J. Reinilä (1879-1951). Dans le même temps, des jardins groupés furent créés à Helsinki, Turku, Oulu, Porvoo et Vyborg.

12 Helsinki a créé son premier jardin familial en 1918 à Ruskeasuo (nom d'origine en suédois Brunakärr koloniträdgård, en finnois Ruskeasuon siirtolapuutarha). Le service d'urbanisme de la Ville d'Helsinki a adopté l'idée des jardins familiaux en même temps que celle d'organiser des jardins à l'anglaise. Ces démarches ont été soutenues par l'urbanistearchitecte Birger Brunila (1882-1979). Ce dernier a contribué à la naissance de tous les jardins familiaux entre les années 1920 et 1940 et a imposé que les jardins familiaux soient inscrits dans le PLU comme parcs permanents et protégés par la loi. C'est ainsi que ces jardins familiaux ont immédiatement acquis un statut renforcé dans la structure des 
villes. De ce point de vue, Helsinki a été en avance sur les autres villes de Finlande et du reste de l'Europe? .

\section{Elisabeth Koch, la « Mère » des jardins familiaux}

La paysagiste Elisabeth Koch (1891-1982) est considérée en Finlande comme la "mère » des jardins familiaux. Elisabeth Koch avait étudié le jardinage dans les écoles de Högvalla et Järvilinna dans les années 1910 ; elle crée son agence en 1920 et travaille pour la Ville d'Helsinki et les jardins familiaux de 1925 à 1955. Avec l'architecte Armas Lindgren (1874-1929), elle dessine notamment la cour-jardin de Vallilan Kone \& Silta Oy à Helsinki, en $1928^{10}$. Les dernières recherches en archives et les entretiens oraux avec les administrateurs de l'époque ont montré qu'Elisabeth Koch, inconnue du public, fut la pionnière et la principale créatrice de jardins familiaux dans les années 1930-1940.

Elle réalise sept jardins familiaux à Helsinki : à Kumpula (1926), Vallila (1931), Kivinokka (1934), Tali (1936), Oulunkylä (1940), Marjaniemi (1946) et Pakila (1948). Elle a planifié les espaces extérieurs du jardin pédagogique de Kumpula, en collaboration avec l'architecte Gunnar Taucher, en $1926^{11}$ et dessiné les plans d'autres jardins familiaux en Finlande (fig. 3). Elle a écrit des guides et des articles sur les jardins d'habitations, publiés dans les revues Trädgårdsodlaren (horticulteur) et Siirtolapuutarha (jardin familial). Son guide intitulé Hemträdgården (Maison \& jardin), publié en 1919, fut réédité plusieurs fois. Elisabeth Koch fut déclarée membre d'honneur de l'Association des jardins familiaux en 1961. Elle prit aussi part à la première commission de l'association de Suomen Puutarhaarkkitehdit - Finlands Trädgårdsarkitekter ry (Paysagistes finlandais) ${ }^{12}$.

Figure 3

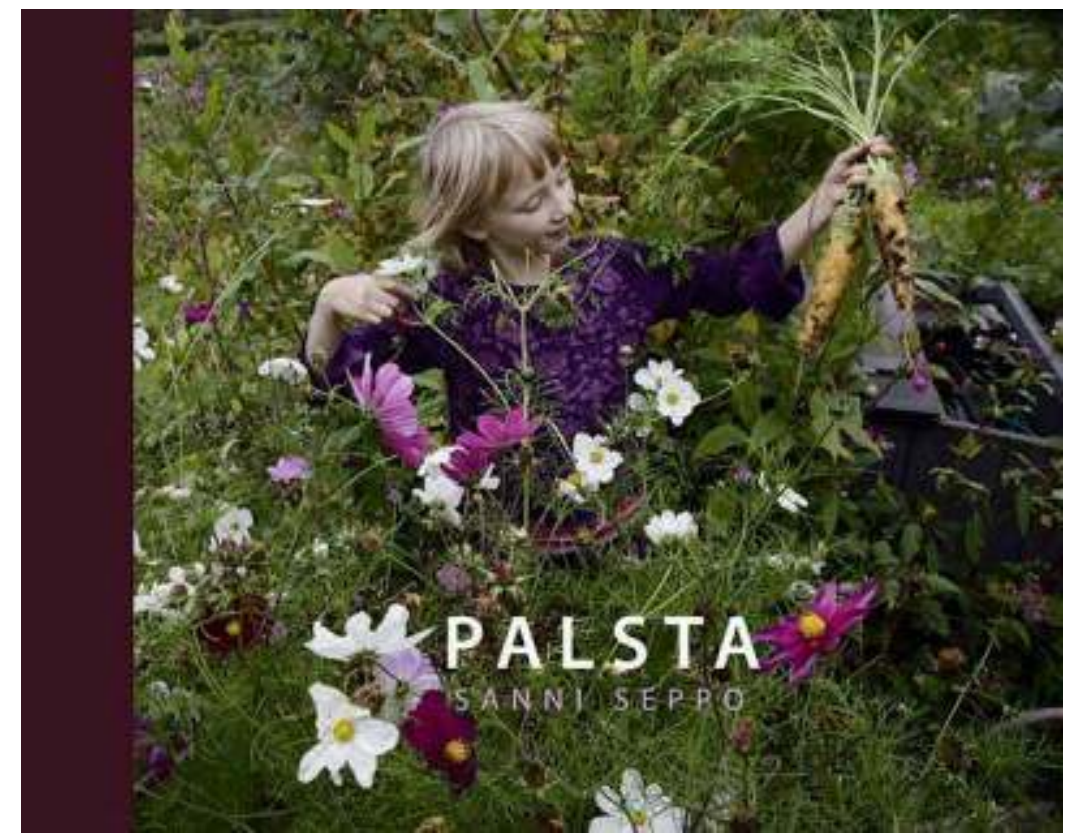

SEPPO, Sanni. Palsta. Maahenki : 2010.

15 Ses idées sur le jardinage urbain sont toujours d'actualité. Dans les jardins familiaux d'Elisabeth Koch, on reconnaît facilement des caractéristiques qui sont sa manière de fabriquer la palette végétale. En effet, certains éléments sont typiques de sa façon de créer: les variétés de végétation et l'utilisation de l'espace. Le manque d'entretien en a 
fait disparaître une partie mais grâce aux récentes recherches, il est possible de les repérer et de les mettre en valeur. Les jardins familiaux de Kumpula, Vallila et Herttoniemi ont été reconnus internationalement dès leur création. Ils illustrent tout le travail emblématique d'Elisabeth Koch: sa façon de comprendre le projet comme une entité complète tout en y associant des détails vernaculaires. Elle a vu les jardins comme une combinaison d'utile et d'esthétique et les a conçus dans le but de créer du bien-être et d'aider les gens dans leur quotidien ${ }^{13}$.

Elisabeth Koch organisait des visites des jardins familiaux et pensait que le travail pratique de sensibilisation des jardiniers amateurs aux bonnes pratiques était un point essentiel. Elle a été la voix des jardins familiaux auprès du public. Elle a diffusé l'idée des jardins familiaux à travers des livrets et des journaux et participait aux séminaires et voyages d'études organisés sur ce sujet à l'étranger ${ }^{14}$. Pour elle, le jardin contribuait à la santé, à la relation avec la nature et à maintenir l'unité familiale. En travaillant dans le jardin, il était possible de créer un lieu de vie, une maison d'été où les membres de la famille pouvaient travailler dehors, faire la récolte, jouer, se reposer ${ }^{15}$.

\section{Formes spécifiques}

17 L'aménagement des jardins familiaux reflète les influences esthétiques de l'époque. Les premiers jardins familiaux ont été planifiés en suivant les principes du jardin géométrique : axes dominants, plantations symétriques et utilisation d'espaces ouverts répétés étaient les éléments majeurs constituant les espaces publics. Ces compositions se retrouvaient également dans les parcelles individuelles. Les limites entre les parcelles et le paysage, entre les espaces privés et publics, sont floues. En Finlande, la relation avec la nature proche est forte. Par exemple, les parcelles d'un jardin familial ou les jardins des maisons individuelles ne sont pas forcément clôturés. Les clôtures, si elles existent, sont le plus souvent faites de végétaux, utilisant des haies ou des arbustes, ou sont construites en bois et couvertes de plantes grimpantes - ce qui reflète bien l'idée de garder le paysage et l'horizon ouverts au regard.

Au début des années 1930, les aires de jeux d'enfants et de sport ont gagné du terrain sur les jardins familiaux ${ }^{16}$. À partir des années 1940, ces derniers ont été maintenus plus librement, en accord avec les idées du modernisme. En vingt ans, «le patrimoine vert » est parvenu à maturité. Les haies sont devenues denses, créant une ambiance intimiste dans les parcelles. La fonction du jardin est restée la même. On y trouvait toujours un jardin potager et un verger. Dans les années 1950, les anciennes parcelles et les parties communes ont été renouvelées dans la plupart des jardins ${ }^{17}$. Après les changements des années 1940, il a fallu attendre jusqu'aux années 1970 pour voir la construction d'un nouveau jardin familial, cette fois-ci à Pakila ${ }^{18}$. Ce n'est ensuite que dans les années 1980 que les jardins familiaux ont acquis leur statut privilégié, toujours en vigueur. La nécessité pure et simple de cultiver de quoi manger a été remplacée par le désir de produire soi-même des aliments sains et de les produire ensemble, en famille, avec ses voisins ou des inconnus, habitants du quartier ou de la même ville ${ }^{19}$.

La planification des jardins familiaux a été mise en œuvre par des fonctionnaires de l'État. Le plan d'urbanisme a été conçu par des architectes-urbanistes, et les plans des parcelles par des paysagistes. Cette organisation avait pour but d'obtenir des entités cohérentes et harmonieuses. Le bail des parcelles comporte l'obligation de les maintenir cultivées et bien entretenues. Cette exigence a été critiquée, car elle limite le choix des végétaux et le 
mode de culture. Le propriétaire se retrouve parfois malgré lui avec un bout de terrain "standardisé » au lieu d'un «jardin de rêve ». Ces règles ne laissent pas les jardiniers exprimer leur inspiration esthétique propre ${ }^{20}$. Toutefois, l'orientation imposée pour des professionnels a été admise comme une directive visant à maintenir un aspect général rationnel et non comme un facteur d'uniformité et de monotonie. Pouvoir choisir la palette végétale ou la couleur de la «maisonnette » et de la clôture en bois donnait la possibilité d'apporter une petite touche personnelle. Cependant, les différentes options sont répertoriées dans un catalogue qui ne laisse donc pas la liberté d'un choix plus large ${ }^{21}$.

20 Il est possible de construire des « maisonnettes » sur les parcelles. Elles servaient d'abris contre la pluie ou de lieux de repos. Elles étaient également utilisées comme cabanes à outils. L'idée de "maisons belvédères» est venue en Finlande de Suède. Traditionnellement, on passait les congés d'été au même endroit, à la campagne, avec un confort minimum (toilettes sèches, sans douche moderne). À l'origine, ces petites constructions en bois comportaient seulement quelques mètres carrés d'espace habitable. Petit à petit, elles sont devenues plus grandes, jusqu'à atteindre $15 \mathrm{~m}^{2}$. L'idée était qu'elles devaient être assez confortables pour y habiter en été, mais pas assez pour permettre d'y habiter tout au long de l'année ${ }^{22}$. Dans les jardins familiaux, le sauna fut souvent la première acquisition majeure.

21 Dans la communauté du jardin familial, les événements marquent les changements de saison: compétitions sportives, stages et fêtes donnent à chaque jardin familial son caractère particulier ${ }^{23}$.

\section{L'héritage des jardins familiaux}

La Fédération finlandaise des jardins familiaux (SSpl) n'a été créée qu'en 1930 et siège à Helsinki. Elle gère 38 associations locales. Aujourd'hui, il existe plusieurs associations de jardins familiaux non affiliées à la Fédération nationale. Le nombre de zones de jardins familiaux atteint presque soixante, dispersées partout en Finlande. Il y a environ 6000 jardiniers associés. De nouveaux lieux naissent toujours. Les jardins partagés les plus importants (9 à Helsinki) sont classés au plan d'occupation des sols (yleiskaava) en tant que lieux remarquables en termes de patrimoine paysager, architectural et culturel. Les grandes villes relèvent le défi d'étendre ces jardins pour répondre à la demande croissante des habitants, tout en pérennisant l'esprit original des lieux. Leur utilisation, leur construction et leur conservation sont aujourd'hui encadrées par plusieurs réglementations en vigueur.

Chaque jardin familial est affilié à une association qui gère la réglementation et toute l'activité dans le jardin. La plupart des jardins se situent sur des terrains loués par la Ville pour laquelle l'association collecte les cotisations payées par chaque propriétaire de parcelle. Dans quelques jardins familiaux, le propriétaire du terrain n'est pas la Ville. Pour donner des exemples, à Mänttä, le jardin familial de Riihossalmi a été créé par le directeur des relations humaines de la société G. A. Serlachius. Cette entreprise loue le terrain à l'association des jardins familiaux. À Nokia, l'association Keho s'est développée sur des terrains appartenant à l'Église protestante. Dès l'origine, les associations se sont donné des règlements et sont à but non lucratif ${ }^{24}$. bien conservé son esprit original ${ }^{25}$. Celui de Herttoniemi est devenu une destination 
touristique grâce à sa situation. Les « maisonnettes " y sont plus grandes $\left(14\right.$ à $\left.16 \mathrm{~m}^{2}\right)$ que celles de Kumpula et mieux équipées. Le jardin de Herttoniemi a été réalisé en suivant les plans originaux dans sa totalité. Il fait partie des espaces verts les plus étendus à l'est $\mathrm{d}^{\prime} H e l s i n \mathrm{ki}^{26}$. Les jardins familiaux d'Oulunkylä, Marjaniemi et Pakila ont également de grandes parcelles à offrir aux particuliers, soit entre 700 et $800 \mathrm{~m}^{2}$, ce qui correspond à la taille d'un jardin d'une maison individuelle traditionnelle. Les jardins familiaux d'Oulunkylä et Pakila, au nord d'Helsinki, font partie du paysage culturel de la rivière de Vantaa.

25 Au début du $\mathrm{xx}^{\mathrm{e}}$ siècle, de nouveaux jardins familiaux ont été construits aux abords des villes. Cependant, l'urbanisation a transformé le paysage dans ces dernières décennies et les constructions se sont étendues sur le territoire. Les jardins familiaux - autrefois situés entre la ville et la campagne - se trouvent maintenant au milieu d'habitations ou de zones commerciales. Ces nouvelles situations créent de nouvelles problématiques, comme la demande croissante des particuliers d'acquérir des parcelles, ou la question du vandalisme envers le mobilier ou les plantations ${ }^{27}$.

Aujourd'hui, ces anciens jardins familiaux sont très populaires. La ville est soumise à la pression contradictoire de créer de nouveaux jardins collectifs et de supprimer ceux qui existent déjà pour libérer des terrains pour des constructions plus rentables. Les jardins familiaux sont inscrits au PLU comme patrimoine protégé - ce qui aide les élus à distribuer les aides pour la maintenance et la protection de ces sites. La liste d'attente pour obtenir une parcelle est longue. La valeur historique des jardins familiaux dans le paysage et la culture finlandaise est bien reconnue. Les "maisonnettes" originelles peuvent se visiter à Hatanpää, Ruskeasuo, Kumpula et Vallila ${ }^{28}$.

\section{Parcelles urbaines}

\section{Historique}

Les parcelles urbaines gagnent du terrain dans les villes depuis 2010. L'intérêt pour l'agriculture ou le jardinage urbain va croissant, et de nouvelles formes de parcelles se créent encore. Toutefois, l'histoire de l'évolution des parcelles urbaines en Finlande reste à écrire. Les sources historiques sont dispersées. Certes, il y a bien quelques livres de photos sur le jardinage dans ces parcelles. Les guides pratiques de jardinage sont publiés par les associations qui organisent également des stages sur ces thèmes. Les réseaux sociaux sont un canal important pour diffuser l'information ${ }^{29}$.

À Helsinki, les premières parcelles urbaines étaient occupées par les immigrés russes. Ils ont transmis leur longue tradition de culture des légumes aux Finlandais. Le " Ryssänkaalimaa » [terrain de choux des Russes] et le parc d'Arcadia ont été mis en culture à la fin du XIX ${ }^{\mathrm{e}}$ siècle. Cette initiative revient à Christian Bohnhof (1822-1881), un jardinier originaire d'Allemagne. Des choux, des herbes et des oignons y étaient cultivés. Cet espace a été détruit lors des travaux de construction de la nouvelle ligne de train vers le port à la fin $\mathrm{du} \mathrm{xx}^{\mathrm{e}}$ siècle $^{30}$. La première parcelle occupée par les habitants a été créée dans un quartier industriel de la ville de Tampere en 1916. La parcelle se trouvait dans un quartier ouvrier, entre les maisons en bois. En comparaison du reste de l'Europe, le jardinage urbain est arrivé tardivement en Finlande. La raison est certainement à trouver dans l'évolution de l'urbanisation en général; en effet, la population habitait encore essentiellement à la campagne au début du $\mathrm{xx}^{\mathrm{e}}$ siècle. 


\section{Formes spécifiques : du sud au nord}

d' thapolku pour le jardinage urbain. Le contrat est renouvelé annuellement. Un pot de cuite ou un sac de jardinage peut suffire à créer un petit jardin potager en ville. À Rovaniemi, le climat n'est pas favorable au jardinage, la durée de croissance des plantes est courte. Néanmoins, l'intérêt pour le jardinage pendant cette courte période d'été est grand, et le premier essai de jardinage urbain a regroupé une vingtaine de participants, aussi bien des jeunes que des retraités.

31 À Helsinki, l'association Dodo utilise un endroit emblématique de la ville pour le jardinage urbain. Depuis 2009, elle cultive ses légumes avec des particuliers dans l'ancien dépôt de trains du quartier de Pasila. Kääntöpöytä regroupe diverses activités sociales, parcelles urbaines, café et lieux pour les ateliers pédagogiques, et l'association organise des événements pour sensibiliser au jardinage urbain et bio depuis 2012. L'idée repose sur la ville ouverte, où les citoyens peuvent s'exprimer et partager leur quotidien. Le centre de Kääntöpöytä est une serre de six mètres de haut, entourée de bacs et de plantes en pots. L'activité de jardinage est expérimentale par nature. Les différentes façons de jardiner sont illustrées de façon pédagogique. Une attention particulière est accordée au recyclage des aliments et à l'agriculture biologique. En même temps, l'association développe avec les jardiniers différents concepts de l'agriculture urbaine sociale. Enfin, le jardin sert de moteur à la recherche de nouvelles formes économiques et écologiques novatrices.

Kalasatama est un quartier de l'ancien port en cours de transformation en quartier d'habitation. Le jardinage urbain y est pratiqué depuis 2010 (fig. 4). 150 sacs, contenant du sable provenant du fond de la mer d'Helsinki, y ont été installés pour les plantations. Il n'y a aucune règle disant quoi ou comment cultiver mais radis, petits pois, salades et lentilles y poussent, comme dans les autres jardins potagers de la ville. 


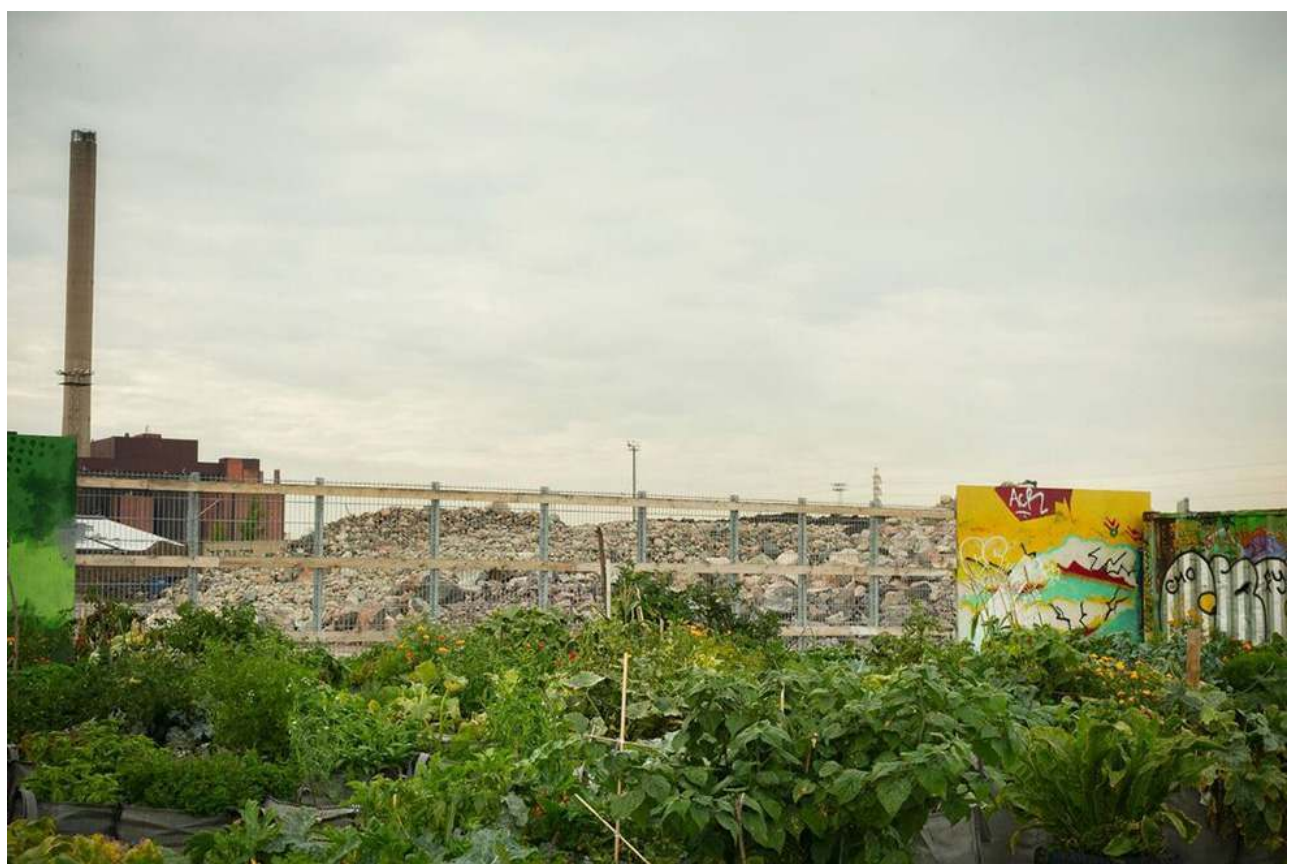

Jardinage urbain à Kalasatama, Helsinki, 2014.

(c) Maija Astikainen.

À Jyväskylä, le jardin de Kangas est uniquement un assemblage de bancs et de sacs de jardinage. Ce jardin a été construit sur un parking, à côté de l'ancien siège de Metsä Group. Les parcelles sont accessibles à tous, aucune cotisation n'est demandée aux participants. En général, en accord avec la politique du jardinage urbain, la priorité est donnée à l'utilisation de matériaux et de produits locaux. Les parcelles urbaines créent un nouveau paysage social et culturel, ouvert et libre pour tout le monde. À l'avenir, selon les dernières recherches, l'intérêt pour le jardinage urbain va probablement encore augmenter. Les essais de plantation d'arbres fruitiers pour les habitants des immeubles ont montré l'intérêt de continuer ces expérimentations. La recherche de variétés suffisamment résistantes et adaptées au climat scandinave s'étend aussi aux autres pays proches $^{31}$.

\section{La situation actuelle}

L'agriculture urbaine a connu un regain d'intérêt parmi toutes les tranches d'âge, y compris chez les jeunes. Le jardinage est pratiqué à la fois sous des formes traditionnelles et nouvelles. Beaucoup de lieux conviennent au jardinage en ville : espaces publics autour des immeubles et des maisons individuelles, jardins partagés, jardins familiaux ainsi que friches et parcs publics. En 2013, cette nouvelle activité a gagné du terrain dans différents lieux à Helsinki. Quatre jardins partagés ont été créés, chacun avec son propre concept, en allant des micro-parcelles aux jardins plantés dans les espaces publics ${ }^{32}$ (fig. 5). 


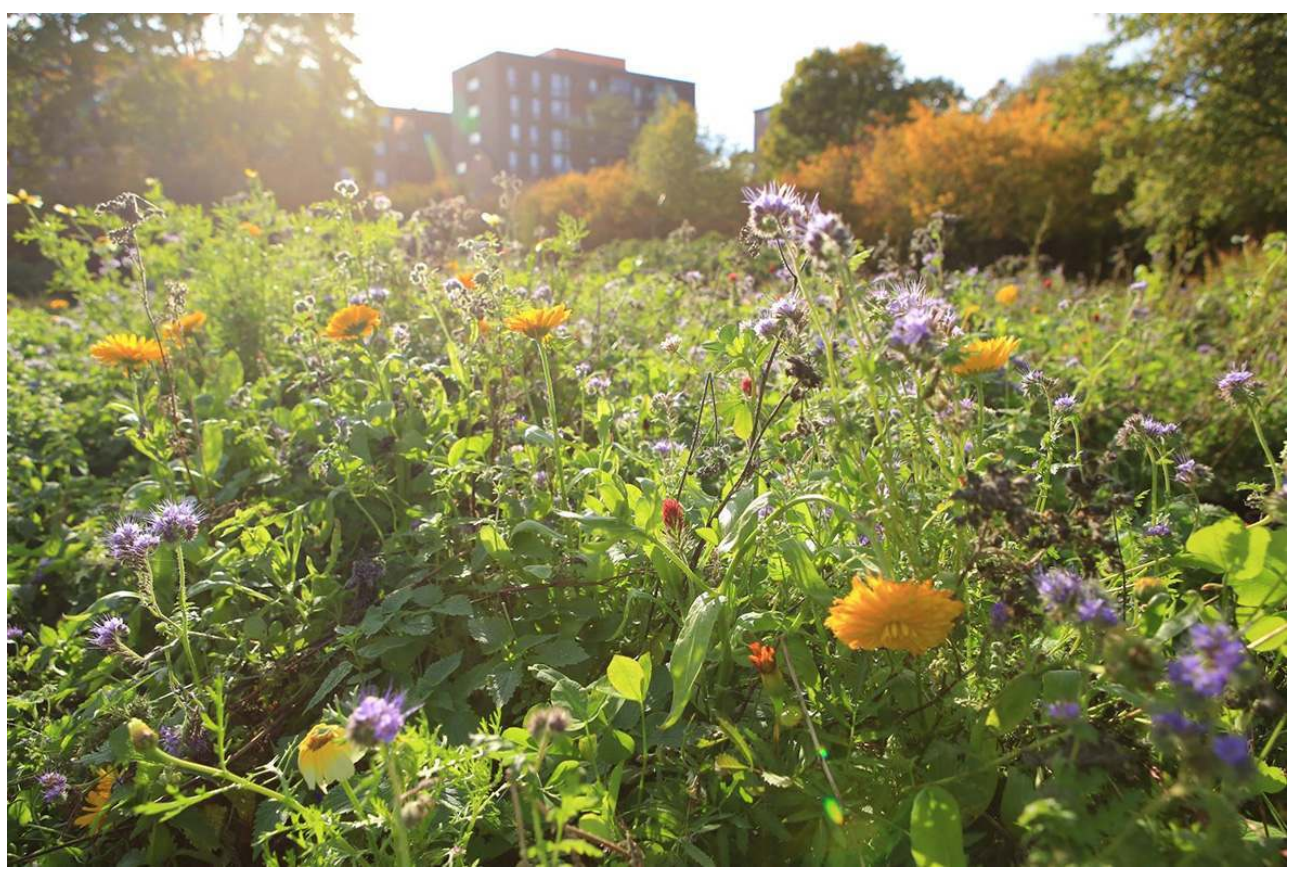

Parcelles urbaines à Hermanni, Helsinki.

(c) Kristiina Salmén.

De nouvelles formes d'agriculture urbaine émergent en continu, et de nouvelles parcelles urbaines sont créées dans des endroits inattendus. Les plantes sont cultivées dans des sacs, des boîtes et des conteneurs facilement transportables. Parfois, en amont de la conception de nouvelles maisons ou d'immeubles, un terrain est réservé au jardinage urbain dans les cours ou sur les toits ${ }^{33}$. Aujourd'hui, on constate également un essor de la plantation d'arbres fruitiers en îlots urbains. C'est une réponse possible pour libérer de nouveaux terrains à cultiver, vu le manque de jardins familiaux pour ceux qui voudraient se lancer dans cette activité. De même, les espaces verts des immeubles d'habitation peuvent servir de lieux au micro-jardinage dans des pots ou jardinières. Les syndics aident à investir dans des arbres fruitiers. Il existe aussi des associations de sensibilisation au jardinage urbain à but non lucratif.

\section{Conclusion : le jardinage innovant, ensemble}

Les jardins familiaux dominent la culture du jardinage en Finlande. De nouvelles idées pour introduire « la nature en ville » ou « un projet innovant, le cinquième élément - des toitures végétalisées dans la ville», ont été lancées pour les années 2011 à 2016. Ce programme intitulé Luomus ( création $)^{34}$ réalise des études scientifiques pour mieux comprendre comment les toitures peuvent être utilisées comme base de culture de la végétation. Les chercheurs espèrent trouver des éléments favorables qui puissent aider à la construction de maisons et d'immeubles plus économiques et plus adaptables à la structure des villes. Par exemple, différents essais sur des toitures en friches, en prairies et en mousses à Helsinki, Vantaa, Lahti, Hollola et Oulu ont montré qu'il était possible d'y créer des biotopes variés, toujours en reliant ces projets aux facteurs socio-économiques et culturels en présence (fig. 6). D'autres formes de jardinage urbain ont également été 
expérimentées, comme le projet de « jardinage urbain et artistique » de l'école supérieure d'Art, de Design et d'Architecture Arabianranta d'Helsinki, en 2011.

\section{Figure 6}

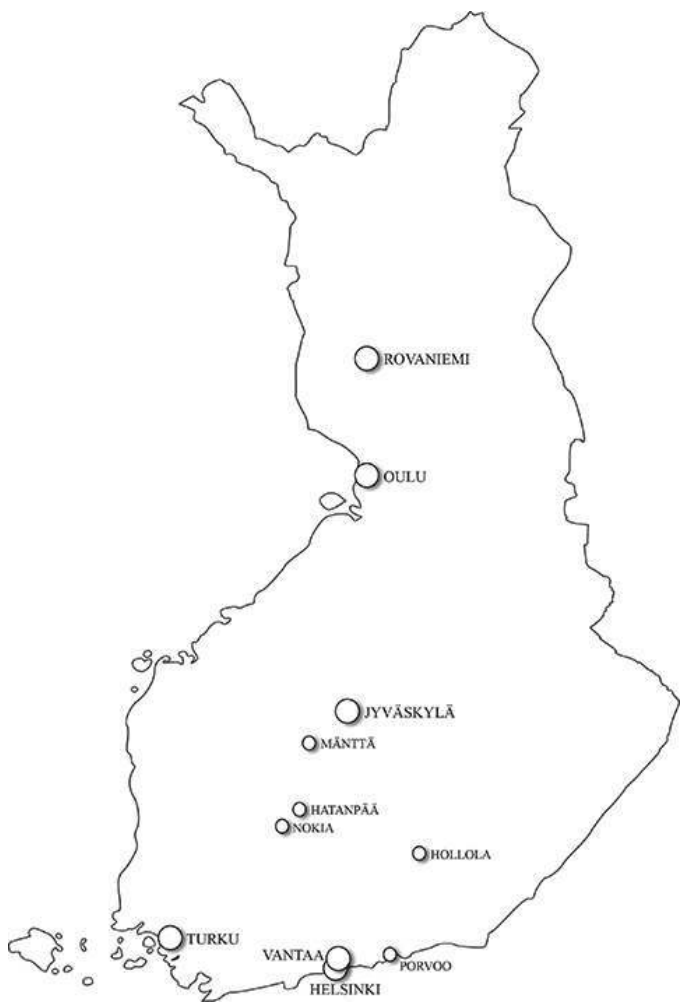

CARTE de LA FINLANDE.

(C) HANNA SORSA.

Les jardins familiaux et les parcelles urbaines font partie du paysage et de la culture urbaine finlandaise. Les dernières recherches ont renforcé leur statut et ont permis d'évoquer la question de leur protection et de leur maintien. Dans le futur, ce pays nordique devrait continuer à développer des formes urbaines spécifiquement conçues pour les jardins familiaux et à innover dans ce domaine.

\section{BIBLIOGRAPHIE}

DAGOGNET, François (dir.). Mort du paysage ? Philosophie et esthétique du paysage. Actes du colloque de Lyon, 1981. Seyssel : Champ Vallon, 1982.

Collectif. Helsingin siirtolapuutarhojen inventointi, Helsinki : Helsingin kaupungin rakennusvalvontavirasto, 2007.

Collectif. Viljellään kaupungissa. Opas yhteisö- ja pienpalstaviljelmien perustamiseen Helsingissä. Helsinki : Helsingin kaupungin rakennusvirasto, 2014/1. 
Collectif. Hedelmäpuita kerrostalojen pihalle, Helsinki : Martat \& Dodo, http://kaupunkiviljely.fi/ wp-content/uploads/2011/04/hedelmapuita_kerrostalopihoille.pdf, 2011 [consulté le 20/11/2017].

Collectif. Luomus. Fifth dimension - Green Roofs and Walls in Urban Areas. Helsinki : Luomus Suomen luonnontieteellinen museo, 2010-1016, https://www.helsinki.fi/en/researchgroups/fifthdimension-green-roofs-and-walls-in-urban-areas [consulté le 15/09/2017].

HEMGÅRD, Gretel. « Kumpulan koulukasvitarha ». Dans Sinkkilä, Jyrki, Donner, Julia, MannerlaMagnusson, Meri (éd.). Unelma paremmasta maailmasta. Moderni puutarha ja maisema Suomessa 1900-1970. Helsinki : Aalto Arts Books, 2016, p. 60-65.

KARISTO, Maria. « Elisabeth Kochin kädenjälki Helsingissä ». Dans Sinkkilä, Jyrki, Donner, Julia, Mannerla-Magnusson, Meri (éd.). Unelma paremmasta maailmasta. Moderni puutarha ja maisema Suomessa 1900-1970. Helsinki : Aalto Arts Books, 2016, p. 112-125.

KARISTO, Maria, KOIVUNEN, Taina, KARISTO, Antti. Kysykää Essiltä! Elisabeth Kochin puutarhat. Helsinki : Maahenki, 2015.

KARISTO, Maria. « Elisabeth Kochin kädenjälki Helsingissä ». Dans Sinkkilä, Jyrki, Donner, Julia, Mannerla-Magnusson, Meri (éd.). Unelma paremmasta maailmasta. Moderni puutarha ja maisema Suomessa 1900-1970. Helsinki : Aalto Arts Books, 2016, p. 112-125.

Collectif. Kääntöpöytä. Helsinki : Dodo, http://kaantopoyta.fi/ [consulté le 19/10/2017].

Collectif. Luomus. Helsinki : Luonnontieteellinen keskusmuseo, https://www.luomus.fi/fi/viidesulottuvuus-viherkatot-osaksi-kaupunkia [consulté le 03/08/2017].

SIIVONEN, Mirja-Riitta, SALONEN, Pirkko, KUCHKA, Tuija. Siirtolapuutarha - kaupunkilaisen puutarha. Helsinki : Tammi, 1999.

SARJAS, Johanna. Yhteisöviljely onnistui Rovaniemellä loistavasti. Helsinki : yle.fi, le $1^{\mathrm{er}}$ juillet 2015 , https://yle.fi/uutiset/3-8252266 [consulté le 18/08/2017].

SARJAS, Johanna, PASSOJA, Annu. Kaupunkiviljely kasvattaa suosiotaan - Rovaniemellä viljellään jo kolmatta kesää. Helsinki : yle.fi, le 2 août 2016, https://yle.fi/uutiset/3-9065053 [consulté le 18/08/2017].

SEPPO, Sanni. Palsta. Helsinki : Maahenki, 2010, p. 168.

\section{NOTES}

1. - DAGOGNET, François (dir.). Mort du paysage? Philosophie et esthétique du paysage. Actes du colloque de Lyon, 1981. Seyssel : Champ Vallon, 1982, p. 28.

2. - KIVILAAKSO, Aura. Puutarha- ja maisema-arkkitehtuuri. Helsinki : Arkkitehtuurimuseo, 2010. Voir le site : http://www.mfa.fi/maisema-arkkitehtuuri [consulté le 22/08/2017].

3. - KARISTO, Maria, KOIVUNEN, Taina, KARISTO, Antti. Kysykää Essiltä! Elisabeth Kochin puutarhat. Helsinki : Maahenki, 2015.

4. - Citation de l'historienne Julia Donner, interview, Helsinki, 15 juillet 2014.

5. - HAUTAMÄKI, Ranja. Portti puutarhaan. Historiallisten puutarhojen inventointiopas. Museoviraston rakennushistorian osaston julkaisuja 21. Helsinki : Edita Oy, 2000.

6. - IVARS, Marja \& LOUNATVUORI, Irma. Puistonäkymiä Museoviraston kartanoissa ja nähtävyyksissä . Museoviraston rakennushistorian osaston julkaisuja 27, 2005. Helsinki, et LISKI, Matti, ALAPETERI, Niina \& RUOFF, Eeva (dir.). Hesperian Esplanadi : puistohistoriallinen selvitys. Helsinki : Helsingin kaupungin rakennusvirasto, katu- ja puisto-osasto 2006. 
7. - Viljellään kaupungissa - opas. Helsinki : Helsingin kaupunki, 2014, p. 6.

8. - SIIVONEN, Mirja-Riitta, SALONEN, Pirkko, KUCHKA, Tuija. Siirtolapuutarha - kaupunkilaisen puutarha. Helsinki : Tammi, 1999, p. 32.

9. - KARISTO, Maria, KOIVUNEN, Taina, KARISTO, Antti. Op. cit., p. 47.

10. - KARISTO, Maria. « Elisabeth Kochin kädenjälki Helsingissä ». Dans SINKKILÄ, Jyrki, DONNER, Julia, MANNERLA-MAGNUSSON, Meri (éd.). Unelma paremmasta maailmasta. Moderni puutarha ja maisema Suomessa 1900-1970. Helsinki : Aalto Arts Books, 2016, p. 112-125.

11. - HEMGÅRD, Gretel. « Kumpulan koulukasvitarha ». Dans ibid., p. 63.

12. - Ibid., p. 251.

13. - KARISTO, Maria, KOIVUNEN, Taina, KARISTO, Antti. Op. cit., p. 175, 179.

14. - Ibid., p. 177.

15. - Ibid., p. 44.

16. - Ibid., p. 47.

17. - Ibid., p. 50.

18. - Ibid., p. 63.

19. - SIIVONEN, Mirja-Riitta, SALONEN, Pirkko, KUCHKA, Tuija. Op. cit., p. 72, 73.

20. - KARISTO, Maria, KOIVUNEN, Taina, KARISTO, Antti. Op. cit., p. 48.

21. - Ibid., p. 49.

22. - SIIVONEN, Mirja-Riitta, SALONEN, Pirkko, KUCHKA, Tuija. Op. cit., p. 131, 133.

23. - Ibid., p. 84.

24. - Ibid., p. 83.

25. - KARISTO, Maria, KOIVUNEN, Taina, KARISTO, Antti. Op. cit., p. 55.

26. - Ibid., p. 60.

27. - SIIVONEN, Mirja-Riitta, SALONEN, Pirkko, KUCHKA, Tuija. Op. cit., p. 136.

28. - Ibid., p. 134.

29. - SARJAS, Johanna. Yhteisöviljely onnistui Rovaniemellä loistavasti. Helsinki : yle.fi, le $1^{\mathrm{er}}$ juillet 2015. Voir le site : https://yle.fi/uutiset/3-8252266 [consulté le 18/08/2017].

30. - Collectif. Viljellään kaupungissa. Opas yhteisö- ja pienpalstaviljelmien perustamiseen Helsingissä. Helsinki : Helsingin kaupungin rakennusvirasto, 2014/1, p. 6.

31. - SARJAS, Johanna, PASSOJA, Annu. Kaupunkiviljely kasvattaa suosiotaan - Rovaniemellä viljellään jo kolmatta kesää. Helsinki : yle.fi, le 2 août 2016. Voir le site : https://yle.fi/uutiset/3-9065053 [consulté le 18/08/2017].

32. - Collectif. Viljellään kaupungissa... Op. cit., p. 6.

33. - KARISTO, Maria, KOIVUNEN, Taina, KARISTO, Antti. op. cit., p. 182.

34. - Voir le site : https://www.luomus.fi/en [consulté le 07/11/2018].

\section{RÉSUMÉS}

Avec l'urbanisation et l'augmentation de la densité de population dans les villes, les jardins partagés ont été créés pour fournir des ressources vivrières, d'abord pour les plus pauvres, puis pour la classe moyenne, avant de se transformer en lieux de détente et de loisirs vers la fin du xxe siècle. Leur utilisation, leur construction et leur conservation sont aujourd'hui encadrées par plusieurs réglementations en vigueur en Finlande. Les plus importants jardins partagés (neuf à Helsinki) sont classés au plan d'occupation des sols (yleiskaava) en tant que lieux remarquables en 
termes de patrimoine paysager, architectural et culturel. Les grandes villes relèvent le défi d'étendre ces jardins pour répondre à la demande croissante des habitants, tout en pérennisant l'esprit original des lieux. Cette contribution a pour objet de présenter les dernières recherches réalisées sur ce sujet en Finlande. Parmi les nombreux articles parus récemment, on peut relever en particulier celui de Maria Karisto sur le travail d'Elisabeth Koch (2015), celui de Gretel Hemgård sur le jardin pédagogique de Kumpula à Helsinki (2016) et celui de Siivonen, Salonen et Kuchka sur les jardins collectifs (1999). Ces articles illustrent les caractéristiques et la problématique de la conservation des jardins partagés d'un point de vue historique. Ils présentent également les paysagistes, humanistes et jardiniers qui ont été à l'initiative des jardins partagés, sans oublier de rendre compte des enjeux spécifiques liés au maintien de ce patrimoine vivant.

With urbanization and the increasing density of housing in cities, community gardens were first used as food resources for the poor, then for the middle classes, before becoming places of relaxation and recreation towards the end of the twentieth century. Nowadays, in Finland, their use, construction and preservation are controlled by several regulations. The most important community gardens - nine in Helsinki - are classified in the "yleiskaava" as outstanding places in terms of landscape, architectural and cultural heritage. Large cities face the challenge of extending these gardens in reply to growing demand from inhabitants, whilst preserving the original spirit of the places concerned. The purpose of this paper is to present the latest research on this topic in Finland. Among many articles published recently, particular attention is paid to that of Maria Karisto on the work of Elisabeth Koch (2015), Gretel Hemgård on the Kumpula education garden (2016) and Siivonen, Salonen and Kuchka on allotment gardens (1999). These articles illustrate the characteristics and the problems concerning the conservation of community gardens from the historical point of view. They also present the landscape gardeners, humanists and gardeners who were at the origins of the community gardens. Finally, the article reports on specific issues relating to the maintenance of this living heritage.

\section{INDEX}

Keywords : family garden, history, heritage, Finland, shared garden, urban land parcel, gardening, urban agriculture, Elisabeth Koch

Mots-clés : jardin familial, histoire, patrimoine, Finlande, jardin partagé, parcelle urbaine, jardinage, agriculture urbaine, Elisabeth Koch

\section{AUTEUR}

HANNA SORSA

MA Landscape Studies, archiviste au bureau des paysages, Gentilly hanna.sorsa@gmail.com 